\title{
Organization Oriented Chemical Computing
}

\author{
Peter Dittrich \\ Friedrich-Schiller University Jena \\ Institute of Computer Science \\ Bio Systems Analysis Group \\ Ernst-Abbe-Platz 14, 07743 Jena, Germany \\ peter.dittrich@uni-jena.de
}

All known life forms process information on a bio-molecular level, which is known to be robust, self-organizing, adaptive, decentralized, asynchronous, fault tolerant, and evolvable. In order to exploit these properties it has been suggested to use artificial chemical systems like P-systems or artificial hormone systems. However, finding the right chemical program appears to be difficult, because computation emerges out of an interplay of many decentralized relatively simple components called molecules. Therefore, a human programmer needs paradigms and tools that allow to predict the behavior of a chemical program, i.e., a set of reaction and transformation rules. In this talk, I focus on how chemical organization theory (Bull. Math. Biol., 69, 1199-, 2007) can help in designing and understanding chemical computing systems. The theory decomposes reaction networks into a hierarchy of closed and self-maintaining sub-networks called organizations. For this analysis stoichiometric information is sufficient, which can be directly obtained from the reaction rules and which is usually independent from kinetics, e.g., a systems state. The fundamental idea of organization oriented chemical programming is to view chemical computing as a movement between organizations (Int. J. Unconv. Comp., 3(4), 285-309, 2007). Our hypothesis is, if the behavior of a chemical program can be explained in terms of organizations, it will be dynamically robust. This hypothesis is supported by a theorem stating that all long-term behavior of a chemical ordinary differential equation will end up in an organization. I will discuss the approach using examples like a chemical XOR, a flip-flop, a controllable oscillator, and the maximum independent set problem, which could be relevant in a distributed sensor networks. Finally, as an open problem, I will discuss how it might be possible to derive chemical organizations directly from implicit reaction rules. 